OPEN ACCESS

Edited by:

Tianyi Yan,

Beijing Institute of Technology, China

Reviewed by:

Li Su,

University of Cambridge,

United Kingdom

Yue Xing,

University of Nottingham

United Kingdom

*Correspondence:

Ying Han

hanying@xwh.ccmu.edu.cn

Shuyu Li

shuyuli@buaa.edu.cn

Received: 19 December 2018 Accepted: 19 February 2019

Published: 22 March 2019

Citation:

Zhao W, Wang $X$, Yin $C$

He M, Li S and Han Y (2019)

Trajectories of the Hippocampal Subfields Atrophy in the Alzheimer's

Disease: A Structural Imaging Study.

Front. Neuroinform. 13:13

doi: 10.3389/fninf.2019.00013

\section{Trajectories of the Hippocampal Subfields Atrophy in the Alzheimer's Disease: A Structural Imaging Study}

\author{
Weina Zhao ${ }^{1,2}$, Xuetong Wang ${ }^{3,4}$, Changhao Yin' ${ }^{2}$, Mengfei $\mathrm{He}^{2}$, Shuyu $\mathrm{Li}^{3,4 *}$ and \\ Ying Han 1,5,6,7*
}

\begin{abstract}
${ }^{1}$ Department of Neurology, Xuanwu Hospital of Capital Medical University, Beijing, China, ${ }^{2}$ Department of Neurology, Mudanjiang Medical University Affiliated Hongqi Hospital, Mudanjiang, China, ${ }^{3}$ School of Biological Science and Medical Engineering, Beihang University, Beijing, China, ${ }^{4}$ Beijing Advanced Innovation Center for Biomedical Engineering, Beihang University, Beijing, China, ${ }^{5}$ Institute of Alzheimer Disease, Beijing Institute for Brain Disorders, Beijing, China, ${ }^{6}$ Institute of Geriatrics, Beijing Hospital, Beijing, China, ${ }^{7}$ National Clinical Research Center for Geriatric Disorders, Beijing, China
\end{abstract}

Background: The hippocampus and hippocampal subfields have been found to be diversely affected in Alzheimer's Disease (AD) and early stages of Alzheimer's disease by neuroimaging studies. However, our knowledge is still lacking about the trajectories of the hippocampus and hippocampal subfields atrophy with the progression of Alzheimer's disease.

Objective: To identify which subfields of the hippocampus differ in the trajectories of Alzheimer's disease by magnetic resonance imaging (MRI) and to determine whether individual differences on memory could be explained by structural volumes of hippocampal subfields.

Methods: Four groups of participants including 41 AD patients, 43 amnestic mild cognitive impairment (aMCl) patients, 35 subjective cognitive decline (SCD) patients and 42 normal controls $(\mathrm{NC})$ received their structural MRI brain scans. Structural MR images were processed by the FreeSurfer 6.0 image analysis suite to extract the hippocampus and its subfields. Furthermore, we investigated relationships between hippocampal subfield volumes and memory test variables (AVLT-immediate recall, AVLT-delayed recall, AVLT-recognition) and the regression model analyses were controlled for age, gender, education and eTIV.

Results: CA1, subiculum, presubiculum, molecular layer and fimbria showed the trend toward significant volume reduction among four groups with the progression of Alzheimer's disease. Volume of left subiculum was most strongly and actively correlated with performance across AVLT measures.

Conclusion: The trend changes in the hippocampus subfields and further illustrates that SCD is the preclinical stage of $A D$ earlier than $\mathrm{aMCl}$. Future studies should aim to associate the atrophy of the hippocampal subfields in SCD with possible conversion to aMCl or AD with longitudinal design.

Keywords: Alzheimer's disease, amnestic mild cognitive impairment, subjective cognitive decline, magnetic resonance imaging, hippocampal subfields 


\section{INTRODUCTION}

The pathophysiological process of Alzheimer's disease (AD) is a neurodegenerative disorder characterized by cognitive decline, which is thought to have begun many years before the diagnosis. With the disease progression, as the preclinical $\mathrm{AD}$, subjective cognitive decline (SCD) have worse cognition than normal controls (NC), while objective examination shows that they have not yet reached the level of amnestic mild cognitive impairment (aMCI) or AD dementia (Molinuevo et al., 2017). The main manifestation of SCD is the decline in memory rather than other domains of cognition. It is formally proposed and standardized by Subjective Cognitive Decline Initiative (SCD-I) in a conceptual framework for research on subjective cognitive decline (Jessen et al., 2014). After adjustment for age, sex and education, the stage of neuropsychological examination below threshold was mild cognitive impairment (MCI) or prodromal AD (Petersen et al., 2018). Subsequently, if there are significant interferences in the ability of work or daily activities, cognitive decline progresses onward to the stage of $\mathrm{AD}$ dementia (Sperling et al., 2011; Jack et al., 2018). These clinical symptoms are caused by the accumulation of pathology leading to the macrostructural disorder of the brain, of which the hippocampus atrophy is the most obvious.

The hippocampus is composed of several subfields with different histological characteristics, rather than a homogeneous structure. Hippocampal atrophy is the most significant structural biomarker of AD imaging (Ritchie et al., 2018). Differential changes in hippocampal atrophy can be relatively easily obtained from magnetic resonance imaging (MRI). The hippocampus and hippocampal subfields are found to be diversely affected in Alzheimer's Disease (AD) and early stages of Alzheimer's disease by neuroimaging studies (de Flores et al., 2015; Chetelat, 2018). The hippocampal atrophy of $\mathrm{AD}$ patients was most significantly involved subiculum and CA1 subfields (Blanken et al., 2017). Other studies have showed that there were more extensive and more evident atrophies in DG/CA3 or subiculum at the lower end of the hippocampus (de Flores et al., 2015). Studies on prodromal AD showed that the focal atrophy of CA1-2 of MCI patients is more obvious than that of normal aging patients (Jessen et al., 2010). The atrophy first appeared in the presubiculum and subiculum of the hippocampus at MCI (Carlesimo et al., 2015). However, SCD subjects are more difficult to identify from the NC because the SCD group showed that the left total hippocampal volume was small with statistically significant difference, while the right total hippocampal volume did not change significantly (van der Flier et al., 2004; Jessen et al., 2006). The atrophy of hippocampal surface is mainly in CA1, and the other regions have obvious overlap with $\mathrm{AD}$ (Perrotin et al., 2017; Evans et al., 2018). The atrophy of the memoryrelated hippocampus and hippocampal subfields is one of the earliest macroscopic features of the trajectories of Alzheimer's disease, and has been reported in autopsies and neuroimaging studies (Braak and Braak, 1991; Frisoni et al., 2008; Mueller et al., 2011; Mak et al., 2017). To our best knowledge, there is little research on the subfield of hippocampus and relationship with memory in SCD.
We hypothesized that there may be 1) a change in the hippocampal subfields at different stages of $\mathrm{AD}$ in accordance to the trajectory of Alzheimer's disease and 2) a relationship between hippocampal subfield volume and memory status (de Flores et al., 2015; Perrotin et al., 2015; Evans et al., 2018). The purpose of this study was to identify which subfields of the hippocampus differ in the trajectories of Alzheimer's disease by magnetic resonance imaging (MRI). In addition, to determine whether individual differences on memory could be explained by structural volumes of hippocampal subfields.

\section{MATERIALS AND METHODS}

\section{Participants}

We prepared 161 right-handed Chinese Han participants including 35 SCD patients, $43 \mathrm{aMCI}$ patients and $41 \mathrm{AD}$ patients, and 42 NC subjects from our databank (NCT: 02225964, 02353845, 02353884, and 03370744). The cognitive functions of all the subjects were assessed by experienced neurologists. Including the Clinical Dementia Rating Scale (CDR) (Morris, 1993), the Chinese version of the Mini-Mental State Examination (MMSE), the Beijing version of Montreal Cognitive Assessment (MoCA) (Lu et al., 2011), the auditory verbal learning test (AVLT) (Guo et al., 2007), an activities of daily living (ADL) assessment, and Hamilton depression rating scale.

The normal controls did not present cognitive decline complaints and their performance in MMSE, MoCA and AVLT were in normal range. The patients with SCD were diagnosed based on the criteria proposed by SCD-I in 2014 (Jessen et al., 2014), including (1) self-reported experience of persistent decline in memory compared to a previous state (within the last 5 years); (2) performance within the normal range on MMSE or MoCA (adjusted for age, sex, and education); (3) the Clinical Dementia Rating (CDR) score is 0 . The patients were diagnosed with aMCI using the Petersen criteria (Petersen, 2004), which have been described in our previous studies (Shu et al., 2018): (a) presence of memory complaint, confirmed by an informant; (b) presence of objective memory impairment measured by MMSE, MoCA and AVLT; (c) failure reach the standard of dementia; (d) CDR score of 0.5. The inclusion criteria for SCD were based on the recent research criteria proposed by National Institute of AgingAlzheimer's Association (NIA-AA) criteria for clinically probable $\mathrm{AD}$ (Sperling et al., 2011): (a) meeting the criteria for dementia; (b) recessive and gradual onset for more than 6 months, not a sudden attack; (c) hippocampal atrophy confirmed by structural MRI; (d) CDR score is equal or greater than 1. Exclusion criteria were prior history of the activities of daily living disorder, stroke, mental disorders, cancer, drug abuse, epilepsy, brain tumors, Parkinson's disease, encephalitis and hypoxic brain damage. All subjects underwent brain MRI examination. The detailed demographic and clinical characteristics of participants are shown in Table 1.

The study approved by the medical research ethics committee and the institutional review board of Xuanwu Hospital, Capital Medical University, Beijing, China. All procedures performed in studies involving human participants were in accordance with 
TABLE 1 | Characteristics of the subjects.

\begin{tabular}{|c|c|c|c|c|}
\hline & $\mathrm{NC}(n=42)$ & $\operatorname{SCD}(n=35)$ & $\operatorname{aMCl}(n=43)$ & $\mathrm{AD}(n=41)$ \\
\hline Age (y) & $64.24 \pm 6.16$ & $64.53 \pm 7.29$ & $67.47 \pm 10.03$ & $68.88 \pm 7.86$ \\
\hline Gender (M/F) & $15 / 27$ & $15 / 20$ & $21 / 22$ & $17 / 24$ \\
\hline Education (y) & $11.17 \pm 0.75$ & $11.83 \pm 0.82$ & $10.44 \pm 0.74$ & $9.68 \pm 0.76$ \\
\hline MMSE & $27.627 \pm 0.530$ & $27.455 \pm 0.582$ & $25.016 \pm 0.520^{+*}$ & $17.782 \pm 0.542^{\#+*}$ \\
\hline MoCA & $25.887 \pm 0.513$ & $24.804 \pm 0.563$ & $17.780 \pm 0.503^{+*}$ & $13.514 \pm 0.524^{\#+*}$ \\
\hline AVLT, immediate recall scores & $9.302 \pm 0.257$ & $8.475 \pm 0.282$ & $5.858 \pm 0.252^{+*}$ & $3.588 \pm 0.263^{\#+*}$ \\
\hline AVLT, delayed recall scores & $10.373 \pm 0.362$ & $8.705 \pm 0.397^{*}$ & $3.226 \pm 0.355^{+*}$ & $1.121 \pm 0.370^{\#+*}$ \\
\hline AVLT, recognition scores & $12.039 \pm 0.464$ & $11.212 \pm 0.509$ & $6.612 \pm 0.455^{+*}$ & $3.450 \pm 0.474^{\#+*}$ \\
\hline
\end{tabular}

ANOVA followed by Bonferroni post hoc analysis for age, education, MMSE, MoCA, CDR and AVLT or the Chi-square test for gender: * $p<0.05$ between NC and SCD, aMCl or AD; ${ }^{+} p<0.05$ between SCD and aMCl or AD. ${ }^{\#} p<0.05$ between aMCl and AD. $n=$ number of subjects; NC, normal control group; SCD, subjectivel cognitive

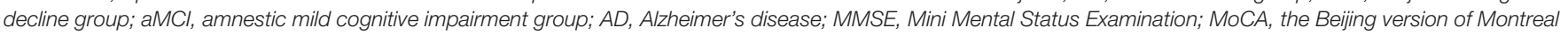
Cognitive Assessment; AVLT, Auditory Verbal Learning Test.

the ethical standards of the institutional and/or national research committee and with the 1964 Helsinki declaration and its later amendments or comparable ethical standards.

\section{Image Acquisition}

The 3T magnetic resonance imaging system (MAGNETOM Trio Tim; Siemens, Erlangen, Germany) was used for image acquisition at the Department of Radiology, XuanWu Hospital, Capital Medical University. T1-weighted MRI scans were acquired at the sagittal plane by using a magnetization prepared rapid acquisition gradient echo sequence with the following parameters: $\mathrm{TR}=1900 \mathrm{~ms}, \mathrm{TE}=2.2 \mathrm{~ms}, \mathrm{FA}=9^{\circ}$, inversion time $(\mathrm{TI})=900 \mathrm{~ms}$, matrix $=256 \times 256$, slices $=176$, thickness $=1.0 \mathrm{~mm}$ and Voxel size $=1 \times 1 \times 1 \mathrm{~mm}^{3}$.

\section{Image Processing}

Structural MR Images were processed by the FreeSurfer image analysis suite, which can be downloaded free of charge from the website (version 6.0.0, http://freesurfer.net/) (Mueller et al., 2018).

First, the entire hippocampal formation was segmented using the routine volumetric FreeSurfer pipeline. Briefly, T1-weighted MR images were corrected for within-subject head motion; then, non-brain tissues were removed using a hybrid watershed/surface deformation algorithm (Segonne et al., 2004). The resulting images were further affine registered to the Talairach space. Subsequently, segmentation of the subcortical and cortical structures (including the hippocampus) was conducted using a probabilistic brain atlas (Fischl et al., 2002). The estimated total intracranial volume (eTIV) of each subject was also calculated using the standard FreeSurfer processing pipeline by exploiting the relationship between the intracranial volume and the linear transformation to the atlas template (Buckner et al., 2004). The eTIV was used to correct for individual differences in head size in the subsequent statistical analysis. Automated segmentation of hippocampal subfields was performed using a built-in module of FreeSurfer, in which a Bayesian statistical model with Markov random field priors was used to estimate the label of each subfield (Van Leemput et al., 2009). This method has been successfully applied to detect hippocampal abnormalities in specific subfields in many neuropsychiatric diseases (Kuhn et al., 2012; Haukvik et al., 2015). A bounding box containing the hippocampus that was upsampled to a $0.5 \mathrm{~mm}$ isotropic resolution was applied to this module. This approach relied on a tetrahedral mesh-based probabilistic atlas of the hippocampal formation, which was constructed from the manual delineation of the right hippocampus based on ultra-high-resolution T1weighted scans $\left(0.38 \times 0.38 \times 0.8 \mathrm{~mm}^{3}\right)$ of 10 normal subjects. By maximizing the posterior probability of a segmentation, the left and right hippocampi were automatically segmented into twelve subfields: hippocampal tail, parasubiculum, presubiculum, subiculum, CA1, CA3, CA4, hippocampus-amygdala transition area (HATA), granule cell layer of dentate gyrus (GC-DG), molecular layer, fimbria, and hippocampal fissure. In this manuscript, the method for automated segmentation is standard. Additionally, the method for segmentation is validated to be accurate by Iglesias et al. (2015). The hippocampal subfield segmentation results are illustrated in Figure 1. The entire hippocampal volume was defined as the sum of the volume of all hippocampal subfields.

\section{Statistical Analysis}

Statistical analysis was carried out using Statistical Package for Social Sciences software (SPSS, version 21.0). All the statistical tests were two-tailed. Categorization of demographic variables was assessed using Chi-square test. Continuous demographic variables were evaluated through ANOVA. In this study, the estimated total intracranial volume (eTIV) was used as a covariate to control head size. Statistically significant differences based on ANOVA $(P<0.05)$ were further explored using Bonferroni post hoc analysis. In the post hoc analysis, the differences between the individual experimental group and the control group were assessed. The left and right hemisphere measurements were analyzed, respectively. In addition, covariance analysis was used to analyze the volume differences in individual hippocampal subfield with age, sex, years of education and eTIV as covariates. Furthermore, we investigated relationships between hippocampal subfield volumes and memory test variables (AVLT-immediate recall, AVLT-delayed recall, AVLTrecognition) through the regression model analyses controlled for age, gender, education and eTIV. 

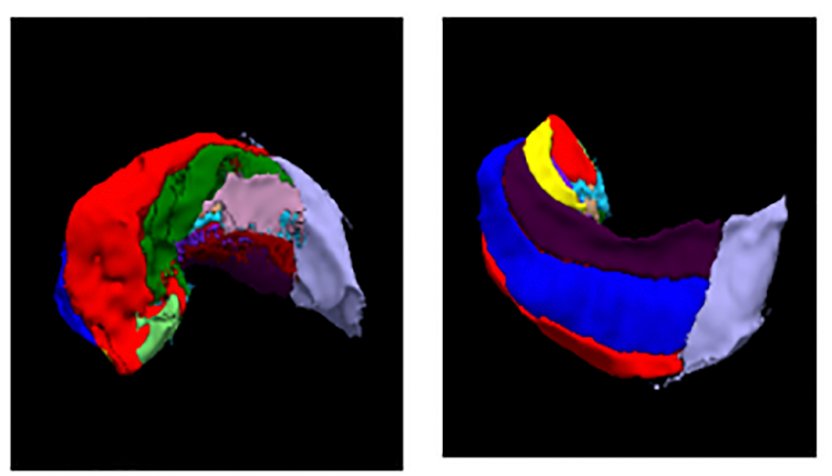

203 parasubiculum

204 presubiculum

205 subiculum

206 CA1

208 CA3

209 CA4

210 GC.ML.DG

211 HATA

212 fimbria

214 molecular_layer_HP

215 hippocampal_fissure

226 HP_tail
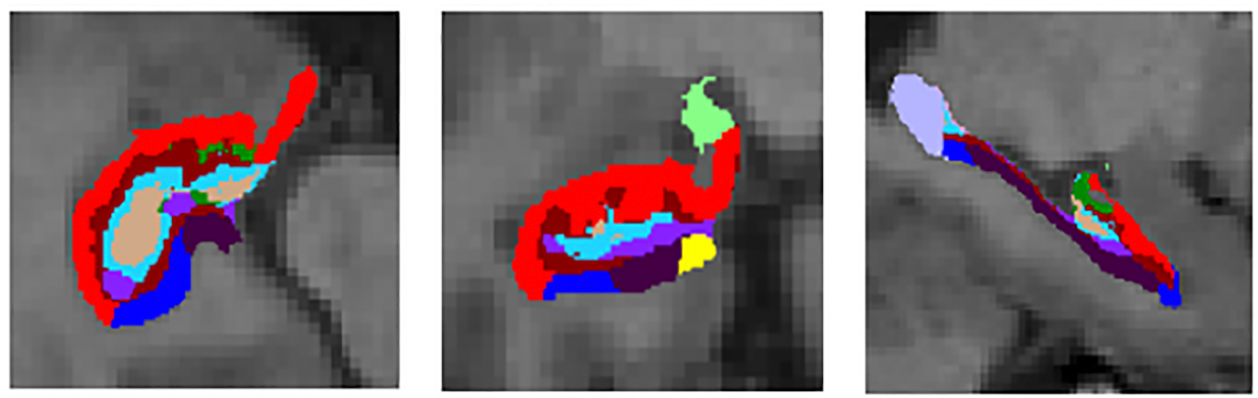

FIGURE 1 | Hippocampal subfield segmentation.

\section{RESULTS}

\section{Demographic Data}

The demographic characteristics of the normal control, the patients of SCD, the patients of aMCI and the patients of $\mathrm{AD}$ are shown in Table 1. Four groups of age, sex, and educational level were well-matched $(P>0.05$ for each group comparison). Comparing SCD and NC groups, there were no significant differences in MoCA, MMSE, immediate recall part of AVLT, the recognition part of AVLT, while significant difference $(P=0.012)$ in the delayed recall part of AVLT. The patients with $\mathrm{AD}$ and $\mathrm{aMCI}$ had significant lower scores in MoCA, MMSE, and AVLT compared with the healthy control participants $(P<0.005)$.

\section{Comparisons of Hippocampal Subregion Volumes}

We tested differences in whole hippocampal volume and all subfields among four groups using ANCOVA with age, years of education, and eTIV as covariates. Table 2 shows the statistical results of hippocampal subfields and hippocampal volumes. The volume of the left whole hippocampus was significantly different between $\mathrm{NC}, \mathrm{SCD}, \mathrm{aMCI}$ and $\mathrm{AD}$ in Figure 2. However, there was no statistically significant difference in the right whole hippocampus between NC and SCD. Compared with $\mathrm{NC}$, aMCI group and $\mathrm{AD}$ group showed significant decreases in right whole hippocampal volume in Figure 3. In addition, the significant decreases were found for SCD and NC in the volume of hippocampal tail, subiculum, presubiculum, molecular layer HP, GC-ML-DG and CA4 of left hippocampal subfields, right presubiculum and fimbria of right hippocampal subfields. Most of the hippocampal subfields showed significant volumetric difference except hippocampal fissure and left parasubiculum between aMCI and NC groups. The significant differences in the hippocampal volume were detected between the $\mathrm{AD}$ and $\mathrm{NC}$ except right hippocampal-fissure. Furthermore, in our study, CA1, subiculum, presubiculum, molecular layer and fimbria showed the trend toward significant volume reduction among four groups with the trajectories of Alzheimer's disease.

\section{Relationship Between AVLT and Hippocampal Subregion Volumes}

In a first step, all potential risk factors (age, education years, sex, GM volume of hippocampal subfields, TIV) were correlated with AVLT scores and only variables correlated with AVLT score at $P<0.2$ were used in subsequent stepwise linear regressions. This was performed to avoid too many independent variables. In the regression model, variables were removed when $P>0.05$. Table 3 presents the results of the linear regression analyses. In our study, volume of left subiculum of all the four groups was most strongly and actively correlated with performance of AVLT three measures.

\section{DISCUSSION}

In this study, we investigated the volumetric difference of hippocampus and hippocampal subregions among $\mathrm{AD}$, 

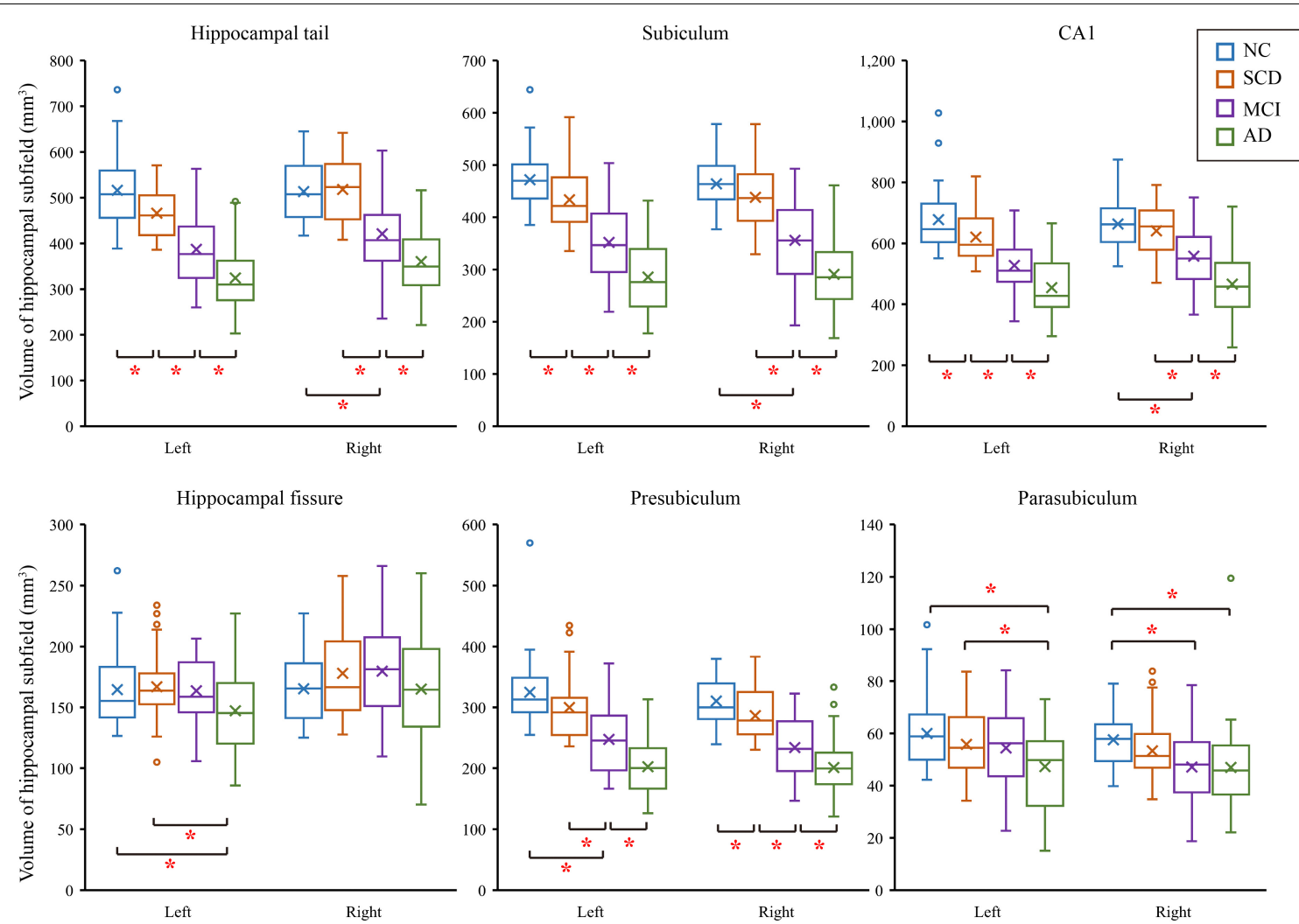

Molecular layer

GC-DG

CA3
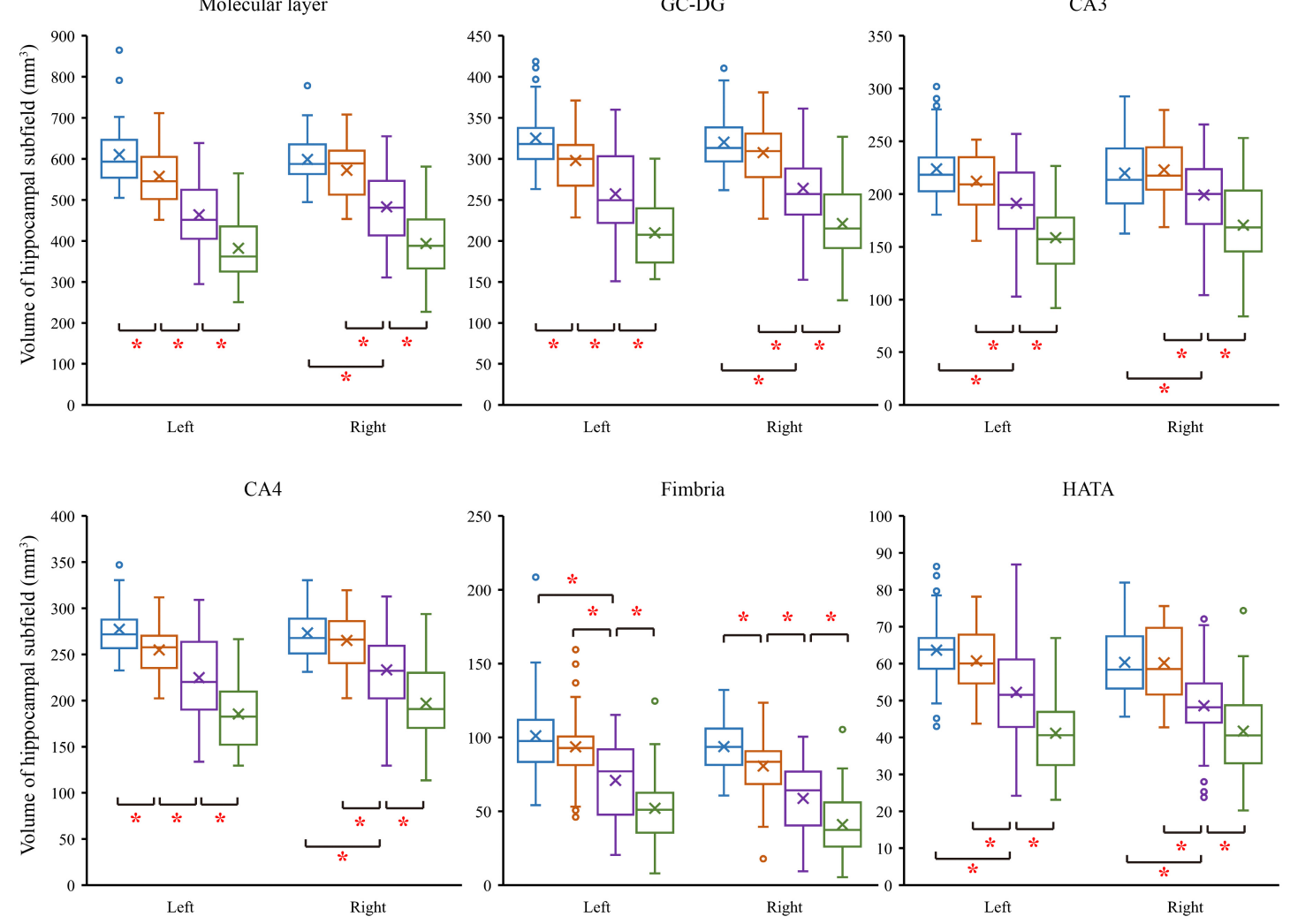

FIGURE 2 | Comparison of hippocampal subregions volume in normal controls and patients with SCD, aMCl and AD. $* P<0.05$. 
aMCI, SCD, and NC subjects. There were also trends in some hippocampal subregions with the trajectories of Alzheimer's disease in addition to the volumetric differences between the four groups. Furthermore, we studied AVLT and typical hippocampal subfields related with memory. It also shown trends with the trajectories of Alzheimer's disease.

In our study, we found that the differences of hippocampus and hippocampal subfields with age, years of education, and eTIV as covariates. The effect of the size of the brain in different subjects was excluded. Our study showed that the difference in volumes was in the left whole hippocampus as that of previous studies (van der Flier et al., 2004; Jessen et al., 2006). We further divided the volume of the hippocampus, and the volumetric subfields of SCD, aMCI and AD were compared with the volumetric subfields of the NC. The hippocampal subfields volume of $\mathrm{AD}$ had significant differences except for right hippocampal fissure. There were also volumetric differences of aMCI in hippocampal tail, subiculum, presubiculum, molecular layer HP, GC-ML-DG, CA4, CA3, fimbria, HATA and right parasubiculum. These were consistent with previous studies (Kang et al., 2018; Su et al., 2018). Previous studies had shown that the volume of the whole hippocampus and hippocampal subfields of SCD and NC were not consistent (van der Flier et al., 2004; Jessen et al., 2006; Carr et al., 2017). But our research found that the volumes of SCD were different from those of NC in left whole hippocampus hippocampal tail, subiculum, presubiculum, molecular layer HP, GC-ML-DG and CA4 of left hippocampal subregions, right presubiculum and right fimbria. Of note, we observed the trend in the CA1, subiculum, presubiculum, molecular layer and fimbria subregions, which were in line with the previous studies, but their studies rarely involved the trajectories of Alzheimer's disease (Perrotin et al., 2015; Carr et al., 2017; Lindberg et al., 2017). The obvious atrophic structures in $\mathrm{AD}$ are located at CA1, subiculum and the presubiculum (Carlesimo et al., 2015). The atrophy of CA1 in MCI has also been reported, which is related to the increased risk of conversion from MCI to AD (Apostolova et al., 2006). In our study, we found that the hippocampus-related subfields had changed as early as SCD stages, however, not all of them showed trend changes. Trend-changing parts are rich in fibers and synapses, which also provide intrahippocampal connections and receive inputs from the hypothalamic lobe and thalamic nucleus. This is strongly correlated with memory impairment in AD patients (Lace et al., 2009). Our finding about the hippocampal volume

TABLE 2 | Comparison of hippocampus and hippocampal subregions volume in normal controls and patients with SCD, aMCI and AD.

\begin{tabular}{|c|c|c|c|c|}
\hline & NC $(n=42)$ & $\operatorname{SCD}(n=35)$ & aMCI $(n=43)$ & $\mathrm{AD}(n=41)$ \\
\hline & Mean $\pm S D$ & Mean $\pm S D$ & Mean $\pm S D$ & Mean $\pm S D$ \\
\hline left_Whole_hippocampus & $3680.289 \pm 66.434$ & $3361.059 \pm 72.289$ & $2783.291 \pm 66.006$ & $2355.177 \pm 67.634$ \\
\hline left_Hippocampal_tail & $517.540 \pm 10.878$ & $466.880 \pm 11.837$ & $383.044 \pm 10.808$ & $326.011 \pm 11.075$ \\
\hline left_subiculum & $474.634 \pm 9.777$ & $434.136 \pm 10.639$ & $346.972 \pm 9.714$ & $287.218 \pm 9.954$ \\
\hline left_CA1 & $685.699 \pm 13.496$ & $622.925 \pm 14.685$ & $517.051 \pm 13.409$ & $456.056 \pm 13.740$ \\
\hline left_hippocampal-fissure & $167.325 \pm 4.356$ & $168.285 \pm 4.740$ & $161.577 \pm 4.328$ & $145.089 \pm 4.434$ \\
\hline left_presubiculum & $326.734 \pm 7.911$ & $300.225 \pm 8.608$ & $243.882 \pm 7.860$ & $203.645 \pm 8.054$ \\
\hline left_parasubiculum & $60.618 \pm 2.092$ & $55.852 \pm 2.276$ & $53.596 \pm 2.079$ & $47.495 \pm 2.130$ \\
\hline left_molecular_layer_HP & $615.260 \pm 11.668$ & $558.698 \pm 12.697$ & $455.821 \pm 11.593$ & $384.814 \pm 11.879$ \\
\hline left_GC-ML-DG & $327.924 \pm 6.251$ & $298.829 \pm 6.802$ & $253.111 \pm 6.211$ & $211.089 \pm 6.364$ \\
\hline left_CA3 & $226.382 \pm 4.893$ & $213.331 \pm 5.325$ & $187.852 \pm 4.862$ & $158.502 \pm 4.982$ \\
\hline left_CA4 & $279.731 \pm 5.250$ & $255.658 \pm 5.713$ & $220.928 \pm 5.217$ & $186.254 \pm 5.345$ \\
\hline left_fimbria & $101.503 \pm 3.972$ & $93.338 \pm 4.322$ & $69.638 \pm 3.946$ & $53.117 \pm 4.044$ \\
\hline left_HATA & $64.264 \pm 1.732$ & $61.186 \pm 1.884$ & $51.397 \pm 1.721$ & $40.976 \pm 1.763$ \\
\hline right_Whole_hippocampus & $3602.039 \pm 63.511$ & $3446.948 \pm 69.108$ & $2852.812 \pm 63.102$ & $2453.308 \pm 64.658$ \\
\hline right_Hippocampal_tail & $515.276 \pm 11.044$ & $517.343 \pm 12.017$ & $415.792 \pm 10.973$ & $364.000 \pm 11.243$ \\
\hline right_subiculum & $467.121 \pm 9.699$ & $438.444 \pm 10.554$ & $349.639 \pm 9.637$ & $293.715 \pm 9.874$ \\
\hline right_CA1 & $670.295 \pm 13.016$ & $641.795 \pm 14.163$ & $546.620 \pm 12.932$ & $470.188 \pm 13.251$ \\
\hline right_hippocampal-fissure & $168.930 \pm 5.345$ & $179.797 \pm 5.816$ & $176.393 \pm 5.311$ & $162.883 \pm 5.442$ \\
\hline right_presubiculum & $311.190 \pm 6.445$ & $285.782 \pm 7.013$ & $231.129 \pm 6.403$ & $203.520 \pm 6.561$ \\
\hline right_parasubiculum & $57.794 \pm 2.095$ & $53.348 \pm 2.280$ & $46.570 \pm 2.082$ & $47.303 \pm 2.133$ \\
\hline right_molecular_layer_HP & $603.151 \pm 11.458$ & $572.299 \pm 12.468$ & $474.457 \pm 11.384$ & $398.113 \pm 11.665$ \\
\hline right_GC-ML-DG & $323.443 \pm 6.254$ & $307.969 \pm 6.805$ & $259.101 \pm 6.213$ & $223.016 \pm 6.367$ \\
\hline right_CA3 & $223.040 \pm 5.422$ & $223.890 \pm 5.900$ & $195.124 \pm 5.388$ & $170.701 \pm 5.520$ \\
\hline right_CA4 & $276.215 \pm 5.430$ & $265.840 \pm 5.908$ & $228.857 \pm 5.395$ & $198.068 \pm 5.528$ \\
\hline right_fimbria & $93.357 \pm 3.230$ & $79.545 \pm 3.514$ & $58.037 \pm 3.209$ & $43.091 \pm 3.288$ \\
\hline right_HATA & $61.156 \pm 1.565$ & $60.693 \pm 1.703$ & $47.486 \pm 1.555$ & $41.594 \pm 1.593$ \\
\hline
\end{tabular}

Mean and standard deviation of subfield and total hippocampal volumes in $\mathrm{mm}^{3}$. 


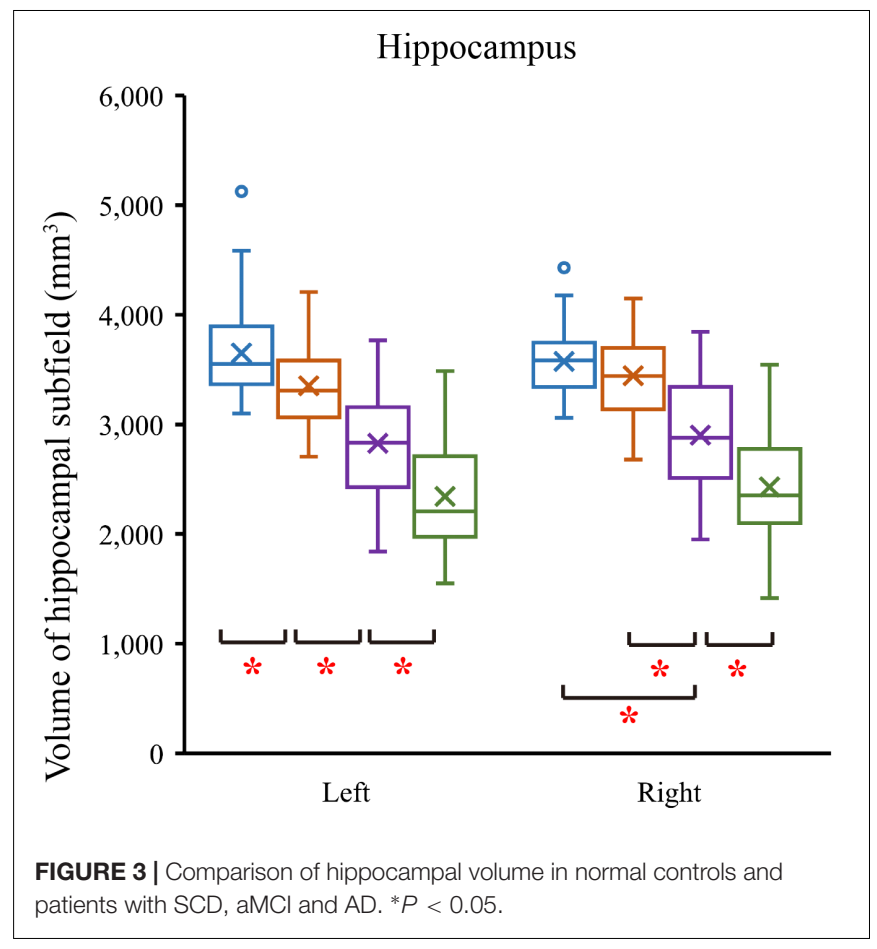

reduction are consistent with neuropathological findings in the progression of AD disease (Mizutani and Kashara, 1995). In our study, the atrophies of CA1, subiculum, presubiculum, molecular layer and fimbria subregions among SCD, aMCI and $\mathrm{AD}$ groups suggest that they may be a potential early biomarker for detecting $\mathrm{AD}$ at the $\mathrm{SCD}$ stage. These results similarly suggest that, compared with normal control subjects, the difference in the volumes of hippocampal subfields and the trend of these changes could show the evolution of $\mathrm{AD}$ in the earlier stage.

The functions of the hippocampal subfield were different, which were related to memory, executive function, attention deficits and so on (Serkova et al., 2016; Evans et al., 2018). The analysis of subfield volumes has been applied to memory neuroscience suggesting that subregion such as CA1, CA3 and dentate gyrus in memory is important (Kesner, 2013; Tamnes et al., 2014; Suthana et al., 2015). In our study, the scores of delayed recalls of AVLT were more closely related to the changes of hippocampal subfields than the score of immediate memory and recognition. As we all knew delayed recalls reflect the episodic memory which was impaired first in AD. Furthermore, the scores of delayed recalls of AVLT were better correlated with left subiculum. It implied that left subiculum might tell diseases earlier as an imaging biomarker (Duara et al., 2012; Jessen et al., 2014; Tamnes et al., 2014; Suthana et al., 2015).

There are limitations in our study. Firstly, the main limitation is the lack of high risk group but asymptomatic control group besides the four groups (AD, aMCI, SCD and NC). In future design, we will collect the high risk but asymptomatic control group. Furthermore, this study was based on cross-sectional data, longitudinal follow-up studies of the same cohort are conducted to identify early imaging markers for disease transformation and prediction. Finally, we only studied hippocampal subregion volume by structural MRI. The combination of the multimodal imaging (i.e., structural, functional MR imaging and positron

TABLE 3 | Linear Regression Models for Different AVLT scores.

\begin{tabular}{|c|c|c|c|c|c|}
\hline Dependent Variable & Variables Included in the Model & Unstandardized $B$ & Coefficients Standard Error & Standardized Coefficients $\beta$ & $\boldsymbol{P}$ \\
\hline & Constant & -3.814 & 0.948 & & $<0.001$ \\
\hline & Left_subiculum & 0.011 & 0.003 & 0.434 & $<0.001$ \\
\hline AVLT, & Sex & 1.239 & 0.301 & 0.248 & $<0.001$ \\
\hline \multirow[t]{3}{*}{ immediate recall scores } & Education years & 0.125 & 0.031 & 0.246 & $<0.001$ \\
\hline & Left_hippocampal tail & 0.007 & 0.002 & 0.272 & 0.007 \\
\hline & Right_p arasubiculum & -0.028 & 0.013 & -0.159 & 0.029 \\
\hline \multirow[t]{6}{*}{ AVLT, delayed recall scores } & Constant & -2.971 & 2.694 & & 0.272 \\
\hline & Left_subiculum & 0.011 & 0.005 & 0.240 & 0.021 \\
\hline & Education years & 0.297 & 0.053 & 0.313 & $<0.001$ \\
\hline & Left_hippocamal_tail & 0.015 & 0.004 & 0.339 & $<0.001$ \\
\hline & TIV & $<0.001$ & 0.000 & -0.153 & 0.006 \\
\hline & Right_fimbria & 0.030 & 0.013 & 0.188 & 0.019 \\
\hline \multirow[t]{6}{*}{ AVLT, recognition scores } & Constant & -5.634 & 1.455 & & $<0.001$ \\
\hline & Left_subiculum & 0.029 & 0.009 & 0.594 & $<0.001$ \\
\hline & Education years & 0.256 & 0.056 & 0.265 & $<0.001$ \\
\hline & Right_fimbira & 0.042 & 0.014 & 0.260 & 0.003 \\
\hline & Left_presubuiculum & -0.025 & 0.011 & -0.370 & 0.025 \\
\hline & Right_hippocamapl_tail & 0.009 & 0.004 & 0.182 & 0.04 \\
\hline
\end{tabular}

In a first step, all potential risk factors (age, education years, sex, GM volume of hippocampal subfields, TIV) were correlated with AVLT scores and only variables correlated with AVLT score at $P<0.2$ were used in subsequent stepwise linear regressions. This was performed to avoid too many independent variables. In the regression model, variables were removed when $P>0.05$. 
emission tomography technique) could be used in our future research.

\section{CONCLUSION}

Our findings show that the trend changes in the hippocampus subfield and further illustrate that SCD is the preclinical stage of $\mathrm{AD}$ earlier than aMCI. The susceptibility of hippocampal subfield to $\mathrm{AD}$ pathological damage is different, so the volume of hippocampal subfield is better than the total volume of hippocampus in identifying early $\mathrm{AD}$. It can better review the trajectory of $\mathrm{AD}$, understand the mechanism, and identify sensitive biological indicators at different stages of $\mathrm{AD}$.

\section{DATA AVAILABILITY}

All datasets generated for this study are included in the manuscript and/or the supplementary files.

\section{REFERENCES}

Apostolova, L. G., Dutton, R. A., Dinov, I. D., Hayashi, K. M., Toga, A. W., Cummings, J. L., et al. (2006). Conversion of mild cognitive impairment to Alzheimer disease predicted by hippocampal atrophy maps. Arch. Neurol. 63, 693-699. doi: 10.1001/archneur.63.5.693

Blanken, A. E., Hurtz, S., Zarow, C., Biado, K., Honarpisheh, H., Somme, J., et al. (2017). Associations between hippocampal morphometry and neuropathologic markers of Alzheimer's disease using 7 T MRI. Neuroimage Clin. 15, 56-61. doi: 10.1016/j.nicl.2017.04.020

Braak, H., and Braak, E. (1991). Neuropathological stageing of Alzheimer-related changes. Acta Neuropathol. 82, 239-259. doi: 10.1007/BF00308809

Buckner, R. L., Head, D., Parker, J., Fotenos, A. F., Marcus, D., Morris, J. C., et al. (2004). A unified approach for morphometric and functional data analysis in young, old, and demented adults using automated atlas-based head size normalization: reliability and validation against manual measurement of total intracranial volume. Neuroimage 23, 724-738. doi: 10.1016/j.neuroimage.2004. 06.018

Carlesimo, G. A., Piras, F., Orfei, M. D., Iorio, M., Caltagirone, C., and Spalletta, G. (2015). Atrophy of presubiculum and subiculum is the earliest hippocampal anatomical marker of Alzheimer's disease. Alzheimers Dement. 1, 24-32. doi: 10.1016/j.dadm.2014.12.001

Carr, V. A., Bernstein, J. D., Favila, S. E., Rutt, B. K., Kerchner, G. A., and Wagner, A. D. (2017). Individual differences in associative memory among older adults explained by hippocampal subfield structure and function. Proc. Natl. Acad. Sci. U.S.A. 114:12075. doi: 10.1073/pnas.1713308114

Chetelat, G. (2018). Multimodal neuroimaging in Alzheimer's disease: early diagnosis, physiopathological mechanisms, and impact of lifestyle. J. Alzheimers Dis. 64, S199-S211. doi: 10.3233/JAD-179920

de Flores, R., La Joie, R., and Chetelat, G. (2015). Structural imaging of hippocampal subfields in healthy aging and Alzheimer's disease. Neuroscience 309, 29-50. doi: 10.1016/j.neuroscience.2015.08.033

Duara, R., Loewenstein, D., Shen, Q., Barker, W., and Varon, D. (2012). Amyloid imaging and hippocampal volumes in the classification of prodromal Alzheimer's disease: the ADNI study. Alzheimers Dement. 8:598. doi: 10.1016/j. jalz.2012.05.2079

Evans, T. E., Adams, H. H. H., Licher, S., Wolters, F. J., van der Lugt, A., Ikram, M. K., et al. (2018). Subregional volumes of the hippocampus in relation to cognitive function and risk of dementia. Neuroimage 178, 129-135. doi: 10 . 1016/j.neuroimage.2018.05.041

Fischl, B., Salat, D. H., Busa, E., Albert, M., Dieterich, M., Haselgrove, C., et al. (2002). Whole brain segmentation: automated labeling of neuroanatomical

\section{AUTHOR CONTRIBUTIONS}

WZ contributed to the main body of the manuscript. YH and SL conceptualized, designed, supervised the study and approved the final version of the manuscript. $\mathrm{CY}$ and $\mathrm{MH}$ contributed to study design and data collection. XW contributed to data analysis and manuscript preparation. All the authors interpreted the data, provided important feedback, and revised the manuscript.

\section{FUNDING}

This article was supported by the National Key Research and Development Program of China (2016YFC1306300), National Natural Science Foundation of China (Grant 61633018, 81430037, 81471731, 31371007, and 81771795), Beijing Municipal Commission of Health and Family Planning (PXM2019_026283_000002), the Mudanjiang Science and Technology Project (Z2018s065), and the Foundation of HongQi (2018HQ-01).

structures in the human brain. Neuron 33, 341-355. doi: 10.1016/S08966273(02)00569-X

Frisoni, G. B., Rossana, G., Elisa, C., Udo, R., Pizzini, F. B., Franco, A., et al. (2008). Mapping local hippocampal changes in Alzheimer's disease and normal ageing with MRI at 3 Tesla. Brain 131, 3266-3276. doi: 10.1093/brain/ awn 280

Guo, Q. H., Sun, Y. M., Pei-Min, Y. U., and Zhen, H. (2007). Norm of auditory verbal learning test in the normal aged in china community. Chin. J. Clin.Psychol. 15, 132-134. doi: 10.3969/j.issn.1005-3611.2007.02.007

Haukvik, U. K., Westlye, L. T., Morch-Johnsen, L., Jorgensen, K. N., Lange, E. H., Dale, A. M., et al. (2015). In vivo hippocampal subfield volumes in schizophrenia and bipolar disorder. Biol. Psychiatry 77, 581-588. doi: 10.1016/ j.biopsych.2014.06.020

Iglesias, J. E., Augustinack, J. C., Nguyen, K., Player, C. M., Player, A., Wright, M., et al. (2015). A computational atlas of the hippocampal formation using ex vivo, ultra-high resolution MRI: application to adaptive segmentation of in vivo MRI. Neuroimage 115, 117-137. doi: 10.1016/j.neuroimage.2015.04.042

Jack, C. R. Jr., Bennett, D. A., Blennow, K., Carrillo, M. C., Dunn, B., Haeberlein, S. B., et al. (2018). NIA-AA research framework: toward a biological definition of Alzheimer's disease. Alzheimers Dement. 14, 535-562. doi: 10.1016/j.jalz. 2018.02.018

Jessen, F., Amariglio, R. E., van Boxtel, M., Breteler, M., Ceccaldi, M., Chetelat, G., et al. (2014). A conceptual framework for research on subjective cognitive decline in preclinical Alzheimer's disease. Alzheimers Dement. 10, 844-852. doi: 10.1016/j.jalz.2014.01.001

Jessen, F., Feyen, L., Freymann, K., Tepest, R., Maier, W., Heun, R., et al. (2006). Volume reduction of the entorhinal cortex in subjective memory impairment. Neurobiol. Aging 27, 1751-1756. doi: 10.1016/j.neurobiolaging.2005.10.010

Jessen, F., Wiese, B., Bachmann, C., Eifflaender-Gorfer, S., Haller, F., Kolsch, H., et al. (2010). Prediction of dementia by subjective memory impairment: effects of severity and temporal association with cognitive impairment. Arch. Gen. Psychiatry 67, 414-422. doi: 10.1001/archgenpsychiatry.2010.30

Kang, D. W., Lim, H. K., Joo, S. H., Lee, N. R., and Lee, C. U. (2018). The association between hippocampal subfield volumes and education in cognitively normal older adults and amnestic mild cognitive impairment patients. Neuropsychiatr. Dis. Treat. 14, 143-152. doi: 10.2147/NDT.S151659

Kesner, R. P. (2013). An analysis of the dentate gyrus function. Behav. Brain Res. 254, 1-7. doi: 10.1016/j.bbr.2013.01.012

Kuhn, S., Musso, F., Mobascher, A., Warbrick, T., Winterer, G., and Gallinat, J. (2012). Hippocampal subfields predict positive symptoms in schizophrenia: first evidence from brain morphometry. Transl. Psychiatry 2:e127. doi: 10.1038/tp. 2012.51 
Lace, G., Savva, G. M., Forster, G., de Silva, R., Brayne, C., Matthews, F. E., et al. (2009). Hippocampal tau pathology is related to neuroanatomical connections: an ageing population-based study. Brain 132(Pt 5), 1324-1334. doi: 10.1093/ brain/awp059

Lindberg, O., Mårtensson, G., Stomrud, E., Palmqvist, S., Wahlund, L. O., Westman, E., et al. (2017). Atrophy of the posterior subiculum is associated with memory impairment, Tau- and $\mathrm{A}^{2}$ pathology in non-demented individuals. Front. Aging Neurosci. 9:306. doi: 10.3389/fnagi.2017.00306

Lu, J., Li, D., Li, F., Zhou, A., Wang, F., Zuo, X., et al. (2011). Montreal cognitive assessment in detecting cognitive impairment in Chinese elderly individuals: a population-based study. J. Geriatr. Psychiatry Neurol. 24, 184-190. doi: 10.1177/ 0891988711422528

Mak, E., Gabel, S., Mirette, H., Su, L., Williams, G. B., Waldman, A., et al. (2017). Structural neuroimaging in preclinical dementia: from microstructural deficits and grey matter atrophy to macroscale connectomic changes. Ageing Res. Rev. 35, 250-264. doi: 10.1016/j.arr.2016.10.001

Mizutani, T., and Kashara, M. (1995). Degeneration of the intrahippocampal routes of the perforant and alvear pathways in senile dementia of Alzheimer type. Neurosci. Lett. 184, 141-144. doi: 10.1016/0304-3940(94)11190-T

Molinuevo, J. L., Rabin, L. A., Amariglio, R., Buckley, R., Dubois, B., Ellis, K. A., et al. (2017). Implementation of subjective cognitive decline criteria in research studies. Alzheimers Dement. 13, 296-311. doi: 10.1016/j.jalz.2016.09.012

Morris, J. C. (1993). The clinical dementia rating (CDR): current version and scoring rules. Neurology 43, 2412-2414. doi: 10.1212/WNL.43.11.2412-a

Mueller, S. G., Chao, L. L., Berman, B., and Weiner, M. W. (2011). Evidence for functional specialization of hippocampal subfields detected by MR subfield volumetry on high resolution images at $4 \mathrm{~T}$. Neuroimage 56, 851-857. doi: 10.1016/j.neuroimage.2011.03.028

Mueller, S. G., Yushkevich, P. A., Das, S., Wang, L., Van Leemput, K., Iglesias, J. E., et al. (2018). Systematic comparison of different techniques to measure hippocampal subfield volumes in ADNI2. Neuroimage Clin. 17, 1006-1018. doi: 10.1016/j.nicl.2017.12.036

Perrotin, A., de Flores, R., Lamberton, F., Poisnel, G., La Joie, R., de la Sayette, V., et al. (2015). Hippocampal subfield volumetry and 3D surface mapping in subjective cognitive decline. J. Alzheimers Dis. 48(Suppl. 1), S141-S150. doi: 10.3233/JAD- 150087

Perrotin, A., La Joie, R., de La Sayette, V., Barre, L., Mezenge, F., Mutlu, J., et al. (2017). Subjective cognitive decline in cognitively normal elders from the community or from a memory clinic: differential affective and imaging correlates. Alzheimers Dement. 13, 550-560. doi: 10.1016/j.jalz.2016.08.011

Petersen, R. C. (2004). Mild cognitive impairment as a diagnostic entity. J. Internal Med. 256, 183-194. doi: 10.1111/j.1365-2796.2004.01388.x

Petersen, R. C., Lopez, O., Armstrong, M. J., Getchius, T. S. D., Ganguli, M., Gloss, D., et al. (2018). Practice guideline update summary: mild cognitive impairment: report of the guideline development, dissemination, and implementation subcommittee of the american academy of neurology. Neurology 90, 126-135. doi: 10.1212/WNL.0000000000004826

Ritchie, K., Carriere, I., Howett, D., Su, L., Hornberger, M., O’Brien, J. T., et al. (2018). Allocentric and egocentric spatial processing in middle-aged adults at high risk of late-onsetalzheimer's disease: the PREVENT dementia study. J. Alzheimers Dis. 65, 885-896. doi: 10.3233/JAD- 180432

Segonne, F., Dale, A. M., Busa, E., Glessner, M., Salat, D., Hahn, H. K., et al. (2004). A hybrid approach to the skull stripping problem in MRI. Neuroimage 22, 1060-1075. doi: 10.1016/j.neuroimage.2004.03.032

Serkova, V. V., Nikol'Skaya, K. A., and Eremina, L. V. (2016). The hippocampus as an organizer of operative attention. Neurosci. Behav. Physiol. 46, 997-1004. doi: 10.1007/s11055-016-0344-4

Shu, N., Wang, X., Bi, Q., Zhao, T., and Han, Y. (2018). Disrupted topologic efficiency of white matter structural connectome in individuals with subjective cognitive decline. Radiology 286, 229-238. doi: 10.1148/radiol.20171 62696

Sperling, R. A., Aisen, P. S., Beckett, L. A., Bennett, D. A., Craft, S., Fagan, A. M., et al. (2011). Toward defining the preclinical stages of Alzheimer's disease: recommendations from the national institute on aging-alzheimer's association workgroups on diagnostic guidelines for alzheimer's disease. Alzheimers Dement. 7, 280-292. doi: 10.1016/j.jalz.2011.03.003

Su, L., Hayes, L., Soteriades, S., Williams, G., Brain, S. A. E., Firbank, M. J., et al. (2018). Hippocampal stratum radiatum, lacunosum, and moleculare sparing in mild cognitive impairment. J. Alzheimers Dis. 61, 415-424. doi: 10.3233/JAD170344

Suthana, N. A., Donix, M., Wozny, D. R., Bazih, A., Jones, M., Heidemann, R. M., et al. (2015). High-resolution 7T fMRI of human hippocampal subfields during associative learning. J. Cogn. Neurosci. 27, 1194-1206. doi: 10.1162/jocn_a_ 00772

Tamnes, C. K., Walhovd, K. B., Engvig, A., Grydeland, H., Krogsrud, S. K., Ostby, Y., et al. (2014). Regional hippocampal volumes and development predict learning and memory. Dev. Neurosci. 36, 161-174. doi: 10.1159/ 000362445

van der Flier, W. M., van Buchem, M. A., Weverling-Rijnsburger, A. W., Mutsaers, E. R., Bollen, E. L., Admiraal-Behloul, F., et al. (2004). Memory complaints in patients with normal cognition are associated with smaller hippocampal volumes. J. Neurol. 251, 671-675. doi: 10.1007/s00415-0040390-7

Van Leemput, K., Bakkour, A., Benner, T., Wiggins, G., Wald, L. L., Augustinack, J., et al. (2009). Automated segmentation of hippocampal subfields from ultrahigh resolution in vivo MRI. Hippocampus 19, 549-557. doi: 10.1002/hipo. 20615

Conflict of Interest Statement: The authors declare that the research was conducted in the absence of any commercial or financial relationships that could be construed as a potential conflict of interest.

Copyright $\odot 2019$ Zhao, Wang, Yin, He, Li and Han. This is an open-access article distributed under the terms of the Creative Commons Attribution License (CC BY). The use, distribution or reproduction in other forums is permitted, provided the original author(s) and the copyright owner(s) are credited and that the original publication in this journal is cited, in accordance with accepted academic practice. No use, distribution or reproduction is permitted which does not comply with these terms. 